\title{
Traffic Adaptive Synchronized Cluster Based MAC Protocol for Cognitive Radio Ad Hoc Network
}

\author{
Sahelee Sultana ${ }^{1}$, Amran Hossain ${ }^{1}$, Md. Obaidur Rahman, ${ }^{1, *}$, and Muzammil Jusoh ${ }^{2}$ \\ ${ }^{1}$ Department of Computer Science and Engineering, Dhaka University of Engineering \& Technology, Gazipur, Bangladesh \\ ${ }^{2}$ School of Computer \& Communication Engineering, Universiti Malaysia Perlis (UniMAP), Perlis, Malaysia
}

\begin{abstract}
In wireless communication, Cognitive Radio Network (CRN) is the contemporary research area to improve efficiency and spectrum utilization. It is structured with both licensed users and unlicensed users. In CRN, unlicensed users also called Cognitive Radio (CR) users are permitted to utilize the free/idle of licensed channels without harmful interference to licensed users. However, accessing idle channels is the big challenging issue due to licensed users' activities. A large number of cluster based MAC protocol have been proposed to solve this issue. In this paper, we have come up with a Traffic Adaptive Synchronized Cluster Based MAC Protocol for Cognitive Radio Ad Hoc Network, with the target of creating cluster structure more vigorous to the licensed users' channel re-occupancy actions, maximize throughput, and minimize switching delay, so that $\mathrm{CR}$ users be able to use the idle spectrum more efficiently. In our protocol, clusters are formed according to Cluster Identification Channel (CIC) and inter-communication is completed without gateway nodes. Finally, we have analysed and implemented our protocol through simulation and it provides better performance in terms of different performance metrics.
\end{abstract}

\section{Introduction}

In wireless communication, wireless users share a small range of radio spectrum in $2.4 \mathrm{GHz}$ to $2.48 \mathrm{GHz}$ and 5.0 $\mathrm{GHz}$ as an unlicensed band. Basically, ISM (Industrial, Scientific, Medical) band is globally fixed and this band is used by all wireless users such as Wireless Local Area Network (WLAN), Wireless Personal Area Network (WPAN), Wireless Sensor Network (WSN), Wireless Body Area Network (WBAN), and Wireless Fidelity (Wi-Fi) Networks comprises with Home networking. It is remarkable that most of the spectrums allocated to television and radio broadcast purposes share a huge amount of bandwidth and are kept idle most of the time; whereas mentioned wireless users share a small range of spectrum [1]. In reality the network gets overcrowded due to the limited number of channels and bandwidth. However, this limited bandwidth would not be adequate to provide better support for the wireless users in terms of Quality of Service (QoS), throughput, energy consumption, load balancing and delay. Thus, a solution is needed to overcome this problem. Cognitive Radio Network (CRN) is one of the probable solutions to overcome this type of problem.

Cognitive Radio (CR) is an auspicious technology for solving the problem of the coexisting of spectrum paucity for new applications [2]. Ultimately, CR is an intellectual transceiver, which can dynamically sense the network environment. It has ability to detect free/idle channels from spectrum and change its transmission parameters dynamically and access the temporally vacant spectrum opportunistically without unbearable interference to primary users (i.e., licensed users) [3].

Cognitive Radio Network (CRN) is an opportunistic network. It is organised with both primary user (PU) also called licensed user and secondary user (SU) also called unlicensed user, in which SUs consist CR-enabled radios and the PUs whose radios need not be CR-enabled [4]. In CRN, SUs are permitted to utilize free of licensed channels without any harmful interference to PUs [5]. In CRN, if two SUs want to communicate, both should active on at least one common channel. So, both the SUs can discover each other, exchange control message and then complete data communication. Since, SUs operates on dissimilar channels based on their channel availability, so channel rendezvous is the main challenging issue in CRN. It is observed that, there are different medium access control (MAC) protocol have been designed for channel rendezvous between two SUs in CRN. Cluster based MAC protocol is one of the popular protocol to rendezvous between to SUs.

Clustering is an operative management methodology in cognitive radio ad hoc networks (CRAHNs) [3]. This technique is used to improve scalability and the performance of CRAHNs. Clustering in CRAHNs involves grouping into clusters and the members of a cluster can communicate with other member of same cluster as well as another member of another cluster. However, in CRN, it is observed that, there are different cluster based approaches exist for channel rendezvous between two CRs. Firstly, in CogMesh approach [6], the authors proposed the neighbour discovery and cluster

\footnotetext{
* Corresponding author: orahman@duet.ac.bd
} 
formation process. In this paper, when a new SU wants to join a cluster, the protocol just permits SU to join the cluster it meets first [6]. However, in this protocol, when $\mathrm{SU}$ is increased in a cluster, overall throughput is decreased and overhead ratio is increased accordingly. Secondly, in Cluster-based MAC approach [7], clustering is formed based on reserved values of neighbour nodes. Reserved values are also computed using three factors such as capacity, stability degree and quality of each link among the neighbours. It is the channel centric and common control channel (CCC) based protocol [7]. However, this approach is comparatively very complex to form cluster and inter cluster communication is impossible due to lack of gateway node(s). In this paper, we emphasis on three special issues, firstly, we propose a new MAC protocol named as Traffic Adaptive Synchronized Cluster Based MAC Protocol for CRAHNs. The core aim of this protocol is to create the cluster construction more robust to PU actions so as to use the free radio spectrum more competently, secondly, we also propose a new cluster formation algorithm based on CIC that deals with the network topology management and maintenance. Therefore, proposed algorithm can maximize the cluster throughput and maintain the cluster stability and thirdly, it significantly improves the performance under high traffic loads.

As a whole our proposed work makes the following contributions:

1. Design a new efficient MAC protocol to create the clusters that work powerfully and also more vigorous to PUs activities and design an efficient cluster formation algorithm based on cluster identification channel CIC (described later).

2. Minimize switching delay of SUs and minimize the contention of same channel and also maximize channel utilization for intra-communication using traffic adaptive technique.

3. Target to maximize throughput and easily build up inter-communication without gateway node(s).

The rest of the paper is organized as follows: in Section 2 the related works and problem statements are presented. Section 3 describes the proposed MAC protocol operation and performance evaluation is described in Section 4, and Section 5 concludes the paper.

\section{Related works and problem statement}

In this section we describe a number of cluster based MAC protocols those have been proposed for CRAHNs to solve the channel rendezvous problem such asCogMesh approach [6], cluster-based MAC approach [7], spectrum opportunity based clustering (SOC) approach [8], stability-capacity-adaptive routing approach [9] and distributed message passing approach [10].

\subsection{CogMesh MAC approach}

CogMesh approach [6], is a decentralized and distributed cluster based MAC protocol.This protocol consists of two periods such as: (i) guaranteed access and random access periods, where the guaranteed access period is used for data communication in and between clusters, and the random access period is used for control message exchange. In CogMesh, clusters are interconnected in two ways such as (i) two Cluster Heads (CHs) are connected by one gateway node, and (ii) $\mathrm{CHs}$ are connected by two gateway nodes when no node is 1-hop neighbor of two CHs [6]. However, in this protocol, if a new SU wants to join a cluster, the protocol just agrees SU to join the cluster it meets first and gateway node must exist for communication. Though, in this protocol, when SU is increased in a cluster, overall throughput is decreased, overhead ratio is increased and switching delay is increased accordingly.

\subsection{Cluster-based MAC approach}

Cluster-based MAC approach [7], is an approach, which main aim is to make the cluster structure more vigorous to PU activities. In this approach, clustering is formed based on available channels, geographical position and experienced statistics and reserved values of neighbour nodes [7]. Reserved values are also computed using three factors such as capacity, stability degree and quality of each link among the neighbours. However, this approach is comparatively very complex to form cluster and inter cluster communication is impossible due to lack of contiguous cluster gateway node(s).

\subsection{SOC approach}

In SOC approach [8], neighbouring SUs are grouped with similar channel availability in the similar cluster. In this approach SUs individually compute their cluster memberships by solving the maximum edge biclique problem [11] and every SU broadcast the computed cluster membership statistics to their neighbors, and update cluster affiliations consequently and then new cluster information is rebroadcasted to SUs. Though, in this protocol generates large number of clusters with small cluster size. For that reason channels switching delay and overhead ratio is increased. In addition intercluster coordination problem may arise, when $\mathrm{CH}$ randomly selects a channel.

\subsection{Stability capacity adaptive routing approach}

Stability capacity adaptive routing approach [9], is a routing scheme for a multi hop high-mobility CRN. This scheme considers the path stability and node capacity. In this protocol, inter-cluster control channels and gateway nodes are selected from the $\mathrm{CHs}$, considering the (i) average delay of control information transmission between two $\mathrm{CHs}$, and (ii) the total throughput of control channels [9]. However, clustering is formed based on the radio link availability. In this approach, $\mathrm{CH}$ and control channel selection is major issue and switching overhead is increased due to PU activities. 


\subsection{Distributed message passing approach}

Distributed message passing approach [10], is distributed clustering approach which is used affinity propagation technique [10]. In this approach, nodes interchange data containing local network information with their direct neighbors until a high excellence set of $\mathrm{CHs}$ and a well-organized cluster structure emerges. Though, in this approach clustering is formed based on affinity dissemination message passing method. Therefore, the result of this approach is, it produce smaller number of cluster with large cluster size. This approach only focus on the clustering structure design, but how to implement them in MAC protocol is not clear.

\section{Proposed cluster-based MAC protocol}

\subsection{System model}

We consider a cognitive radio ad hoc network with $N$ number of channels and there are a total of $M$ SUs in the network and each SU is equipped with a single radio. It is assumed that the radio can be used for listening to the control signals in a Common Control Channel of ISM band, and on the other hand while transmitting/receiving data the SU can have an access to the other available channels. At a given time, a SU can only sense the radio spectrum and generates the Available Channel List $(A C L)$. A node can communicate with its neighbours when they are regulated to the same channel. For $\mathrm{i}^{\text {th }} S U$, denoted by $S U_{i}$, the set of available channel list sensed by $S U_{i}$ is $C_{i}=\left\{\mathrm{ch}_{1}, \mathrm{ch}_{2}, \mathrm{ch}_{3} \ldots \mathrm{ch}_{\mathrm{n}}\right\}$, where $n$ is the total number of available channels detected by $S U_{i}$. Here, we assume that the node mobility is slow and the channel accessibility at each node variations at a relatively low rate, such that the network topology does not alter during the cluster creation process [7].

\subsection{Protocol operation}

In this section we describe the proposed protocol operation. Our proposed protocol operation is divided into a sequence of periods those combinely defines a superframe. The periods of the proposed superframe are (1) Spectrum Sensing Period (2) Beacon \& Cluster Formation Period (3) Data Exchange Period. The arrangement of the superframe is shown in Fig.1.

\begin{tabular}{|l|l|l|}
\hline Spectrum & Beacon \& & Data Exchange Period (3) \\
Sensing & Cluster & \\
Period & Formation & \\
$(1)$ & Period (2) & \\
\hline
\end{tabular}

Fig. 1. Superframe Structure

The description of sequences are described in following sections. It is to note the superframe consists of a fixed part having the periods 1 and 2 and a dynamic part with period 3. The duration of dynamic periods depend on the number of available channels $n$ amongst all the nodes.

\subsubsection{Spectrum Sensing and Available Channel List Sorting}

Initially, when a SU appears in the network, the node scan the channel spectrum and upon detecting available channels creates an Available Channel List (ACL). Thereafter, sort the $A C L$ in ascending order, which is mandatory for cluster formation. Suppose after spectrum sensing, nodes 1, 2, 3, 4, 5 and 6 determine $\left\{\mathrm{ch}_{3}, \mathrm{ch}_{1}, \mathrm{ch}_{2}\right\},\left\{\mathrm{ch}_{1}, \mathrm{ch}_{5}, \mathrm{ch}_{2}\right\},\left\{\mathrm{ch}_{2}, \mathrm{ch}_{3}\right\},\left\{\mathrm{ch}_{5}, \mathrm{ch}_{4}\right\},\left\{\mathrm{ch}_{5}, \mathrm{ch}_{3}\right\}$ and $\left\{\mathrm{ch}_{4}, \mathrm{ch}_{3}, \mathrm{ch}_{5}\right\}$ as available channels, respectively and after sorting, the sorted $A C L$ of nodes 1,2, 3, 4, 5 and 6 will be $\left\{\mathrm{ch}_{1}, \mathrm{ch}_{2}, \mathrm{ch}_{3}\right\},\left\{\mathrm{ch}_{1}, \mathrm{ch}_{2}, \mathrm{ch}_{5}\right\},\left\{\mathrm{ch}_{2}, \mathrm{ch}_{3}\right\},\left\{\mathrm{ch}_{4}\right.$, $\left.\mathrm{ch}_{5}\right\},\left\{\mathrm{ch}_{3}, \mathrm{ch}_{5}\right\}$ and $\left\{\mathrm{ch}_{3}, \mathrm{ch}_{4}, \mathrm{ch}_{5}\right\}$ respectively. We have defined the first channel of the sorted $A C L$ as Cluster Identification Channel (CIC). So, $\mathrm{ch}_{1}$ will be the $C I C$ of nodes 1 and $2, \mathrm{ch}_{2}$ will be the $C I C$ of node $3, \mathrm{ch}_{3}$ will be the $C I C$ of nodes 5 and 6 , and $\mathrm{ch}_{4}$ will be the $C I C$ of node 4 respectively.

\subsubsection{Beacon Period in Common Control Channel (CCC)}

After spectrum sensing, all the SUs tune their radio to a Common Control Channel (CCC) and we assume that the CCC would be a frequency channel from the unlicensed band. The motivation of using the $\mathrm{CCC}$ in unlicensed band is due to its always anytime availability, since there is no chance of occupying the CCC by the PU at any instance. Therefore, the CCC can be used for the beacon period of the superframe (as mentioned in Fig. 1). Furthermore, in the beacon period all the nodes of the network synchronize their clocks [12], exchange their control messages and share their respective ACLs. Hence, all the nodes of the network become aware about its corresponding cluster and cluster neighbours.

\subsubsection{Cluster formation}

According to our cluster formation algorithm, at most number of cluster would be $N$. Here, $N$ is the total number of channels. Consider a network with 15 SUs, 6 PUs and total channel number $N=6$ as shown in Fig. 2 . The nodes sorted $A C L$ is shown in table 1 . In the cluster formation algorithm, according to $C I C$, the network is divided into 4 clusters. So, nodes $8,14,4,5$, and 15 will form cluster-1(i.e., $C I C=1$ ), nodes $11,1,2,3$ and 10 will form cluster-2 (i.e., $C I C=2$ ), nodes 6,7 , and 9 will form cluster-3(i.e., $C I C=3$ ), and nodes 12 and 13 will form cluster-4 (i.e., $C I C=4$ ), which is shown in Fig. 3.

\subsubsection{Node join to cluster or re-clustering}

In this section we discuss (i) how a new node joins to the network as well as to cluster (ii) how handle the PU activities if a PU is occupied any channel (iii) how handle the $\mathrm{PU}$ activities if $\mathrm{PU}$ is occupied the CIC. 
Table 1. Sorted ACL of all nodes.

\begin{tabular}{|c|c|}
\hline $\begin{array}{l}\text { No. of } \\
\text { Nodes }\end{array}$ & $\begin{array}{l}\text { Sorted } \\
\text { (ACL) }\end{array}$ \\
\hline 1 & $\left\{\mathrm{ch}_{2}, \mathrm{ch}_{3}, \mathrm{ch}_{5}, \mathrm{ch}_{6}\right\}$ \\
\hline 2 & $\left\{\mathrm{ch}_{2}, \mathrm{ch}_{3}, \mathrm{ch}_{5}, \mathrm{ch}_{6}\right\}$ \\
\hline 3 & $\left\{\mathrm{ch}_{2}, \mathrm{ch}_{3}, \mathrm{ch}_{5}, \mathrm{ch}_{6}\right\}$ \\
\hline 4 & $\left\{\mathrm{ch}_{1}, \mathrm{ch}_{3}, \mathrm{ch}_{5}, \mathrm{ch}_{6}\right\}$ \\
\hline 5 & $\left\{\mathrm{ch}_{1}, \mathrm{ch}_{2}, \mathrm{ch}_{3}, \mathrm{ch}_{4}, \mathrm{ch}_{6}\right\}$ \\
\hline 6 & $\left\{\mathrm{ch}_{3}, \mathrm{ch}_{5}, \mathrm{ch}_{6}\right\}$ \\
\hline 7 & $\left\{\mathrm{ch}_{3}, \mathrm{ch}_{5}, \mathrm{ch}_{6}\right\}$ \\
\hline 8 & $\left\{\mathrm{ch}_{1}, \mathrm{ch}_{3}, \mathrm{ch}_{4}, \mathrm{ch}_{5}\right\}$ \\
\hline 9 & $\left\{\mathrm{ch}_{3}, \mathrm{ch}_{4}, \mathrm{ch}_{5}, \mathrm{ch}_{6}\right\}$ \\
\hline 10 & $\left\{\mathrm{ch}_{2}, \mathrm{ch}_{3}, \mathrm{ch}_{4}, \mathrm{ch}_{5}, \mathrm{ch}_{6}\right\}$ \\
\hline 11 & $\left\{\mathrm{ch}_{2}, \mathrm{ch}_{4}, \mathrm{ch}_{5}, \mathrm{ch}_{6}\right\}$ \\
\hline 12 & $\left\{\mathrm{ch}_{4}, \mathrm{ch}_{5}, \mathrm{ch}_{6}\right\}$ \\
\hline 13 & $\left\{\mathrm{ch}_{4}, \mathrm{ch}_{5}, \mathrm{ch}_{6}\right\}$ \\
\hline 14 & $\left\{\mathrm{ch}_{1}, \mathrm{ch}_{2}, \mathrm{ch}_{3}, \mathrm{ch}_{4}, \mathrm{ch}_{5}\right\}$ \\
\hline 15 & $\left\{\mathrm{ch}_{1}, \mathrm{ch}_{3}, \mathrm{ch}_{4}, \mathrm{ch}_{6}\right\}$ \\
\hline
\end{tabular}

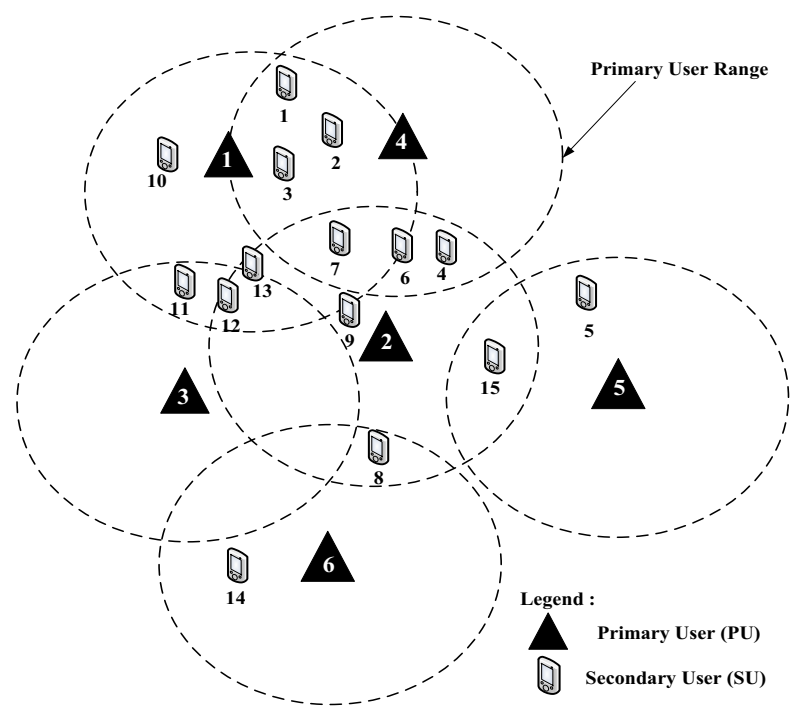

Fig. 2. Simple CRN architecture model

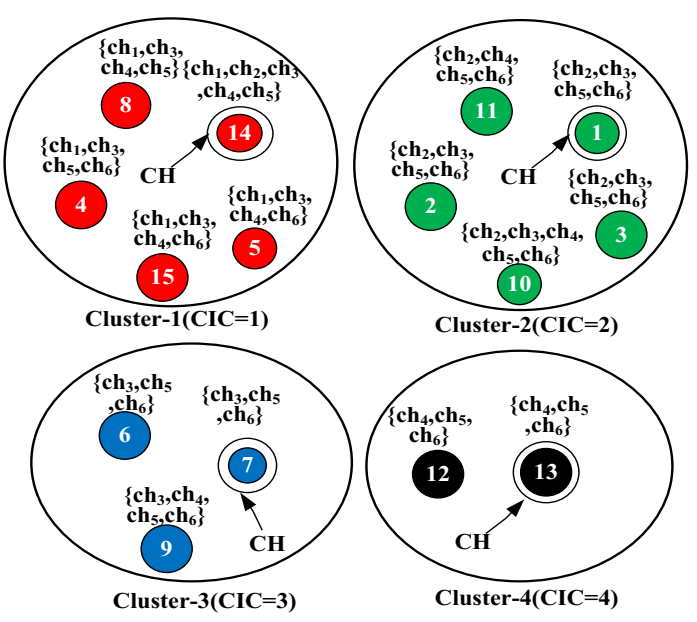

Fig. 3. Clusters after cluster formation

Firstly, when a new node wants to join to a cluster, it sends beacon on CCC and it will join a cluster according to CIC. Secondly, if a PU is occupied any channel instead of $C I C$ or any occupied channel is free at any time. This means that when the sorted $A C L$ at a node changes due to the PU, the node's neighbours have to be updated about its new channel list. Thirdly, if a PU is occupied CIC of a node(s) in same cluster. Then the node(s) resorted its $A C L$, and then it will join to that cluster according to new $C I C$ and it is maintained by $\mathrm{CH}$. Suppose in Fig.3, node 4 is the member of cluster-1 and its sorted $A C L$ is $\left\{\mathrm{ch}_{1}, \mathrm{ch}_{3}, \mathrm{ch}_{5}, \mathrm{ch}_{6}\right\}$ so it's $C I C$ is $\left\{\operatorname{ch}_{1}\right\}$. Let a $P U$ occupied the $C I C$ of node 4 then the new sorted $A C L$ of node 4 is $\left\{\mathrm{ch}_{3}, \mathrm{ch}_{5}, \mathrm{ch}_{6}\right\}$, then the node 4 wants to join to the cluster-3, because its new $C I C$ is now 3. Therefore, 4 sends the beacon on CCC and exchange its information with its neighbours. In this case, the $C H$ of cluster- 3 response the beacon of 4 and finally joint to the cluster-3. This procedure is called reclustering. So the node 4 will be the new member of cluster-3.

\subsubsection{Data Exchange Period}

Data exchange period is dynamic part of our proposed protocol. It is called dynamic because the duration of dynamic periods depend on the number of available channels $n$ amongst all the nodes. The node(s) divides time into $n$ number of identical time slots of fixed duration ' $T_{C}$ '. Here $n$ is the number of available channels of a node. Each time slot is dedicated to one channel for data exchange. The time slots of a node may vary according to its channel availability. Hence, number of channel slot $\left(N_{\text {slot }}\right)$ of a node is dynamic, which is depends on available channels $n$, so channel slots of a node at most $N_{c h}$ slots. Therefore, number of channel slots of a node must be $N_{\text {slot }} \leq N_{c h}$. Data communication between nodes may be two types such as (i) Intracommunication and (ii) Inter-communication. The operations are described below.

\subsubsection{Intra-communication}

When nodes of same cluster are communicating with each other, this is called intra-communication. During

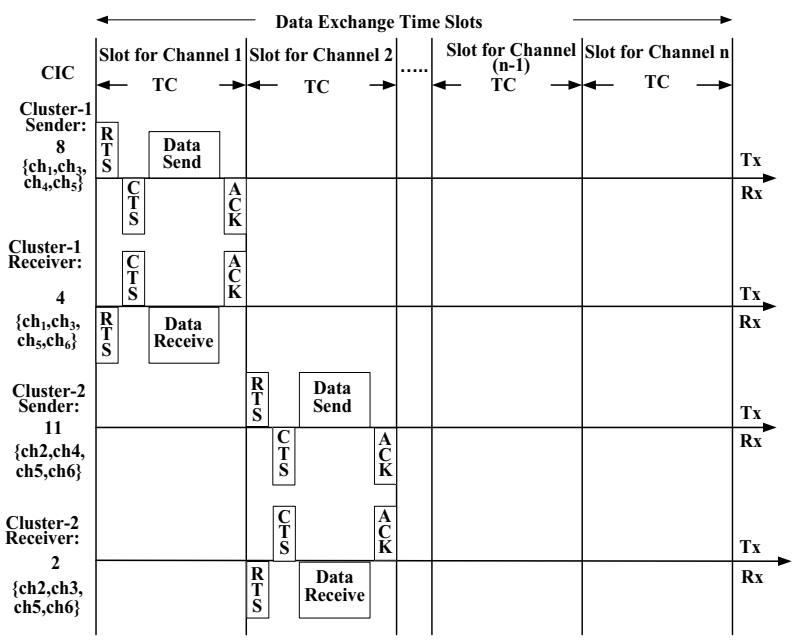

Fig. 4. Intra-communication scenario 
intra-communication, a node transmits and receives data packets on $C I C$, which is available on all nodes of the same cluster. Since each time slot is dedicated to one channel. However, in case of increasing the traffic load on $C I C$, then nodes exchange data packets on second available channel in sorted $A C L$, which is maintained by $\mathrm{CH}$. The details intra-communication is illustrated in Fig. 4. Suppose according to Fig. 3 cluster-1 has five nodes $8,4,14,15$, and 5 and $C I C$ is $\left\{\operatorname{ch}_{1}\right\}$. In Fig. 4, let, a sender node 8 wants to communicate with receiver node 4 , then 8 will start exchange control signals (i.e., $R T S$ and CTS) similar to IEEE 802.11 DCF with node 4 on their $C I C$ (i.e., $\mathrm{ch}_{1}$ ) in time slot 1 , then 8 sends data packets to node 4 and node 4 receives data packets from node 8 and finally node 4 sends acknowledgement (i.e., ACK) to node 8 for confirming successful communication. However, according to this procedure other cluster members are completed their communication on their CIC during intracommunication.

\subsubsection{Inter-communication}

When members of one cluster are communicating with members of other clusters, this is called intercommunication. It is also called cluster to cluster communication. Since, all nodes are synchronized and each node aware the information's about its neighbours and their sorted $A C L$, so during inter-communication, nodes transmits and receives data packets on particular time slot, which is assigned to a particular channel and available of both of them. Inter-communication is illustrated in Fig. 5. In Fig. 5, suppose according to Fig. 3 sender node 14 of cluster- 1 wants to communicate with receiver node 11 of cluster-2. Then node 14 chooses one of the channels common between itself and its neighbour. Then node 14 waits for the time slot which represents the chosen channel. Since all nodes will be listening to that channel in that slot duration, then 14 will start exchange control signals (i.e., RTS and CTS) with its neighbour node 11 and data is exchanged after exchanging the control signals similar to IEEE 802.11 DCF and so on.

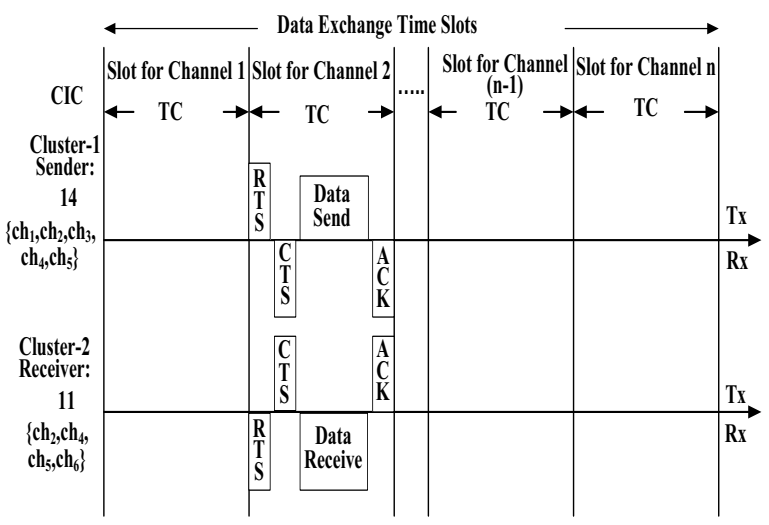

\section{Performance evaluation}

We have demonstrated the performance evaluation and implemented of our proposed protocol and compare the results with that of a Custer-based MAC protocol. We have used our own simulation model developed in Java platform. In this simulation environment, 10 channels are used for modeling licensed channels for PUs and randomly some of the channels made free/unlicensed and set available to the SUs. Maximum 100 nodes are deployed in $600 \times 600 \mathrm{~m}^{2}$ area to make variation in number of PU and SU. The transmission range of each node is set to $250 \mathrm{~m}$. In our simulation, for evaluation the performance of proposed MAC protocol, and Clusterbased MAC, we have considered three performance metrics such as-, Number of Cluster, Throughput, Packet Transmission Delay.

\subsection{Number of cluster formation}

Fig. 6 shows the number of cluster formation of proposed MAC with the Cluster-based MAC protocols as the number of SUs are increased. From the Fig. 6 we can see that as the number of SUs nodes increases, the Cluster-based MAC protocol generates a large number of clusters. The main cause for this behaviour is that Cluster-based MAC is used link centric cluster formation algorithm, and on the other hand our proposed MAC protocol is used number of channel centric cluster formation algorithm. Cluster-based MAC uses multiple $\mathrm{CCC}$, so increase number of cluster, increase the complexity as well as decrease channel rendezvous. In contrast, our proposed MAC does not use multiple CCC, it uses only CIC, so decrease number of cluster and it is steady which is shown in Fig. 6 as worst case and average case.

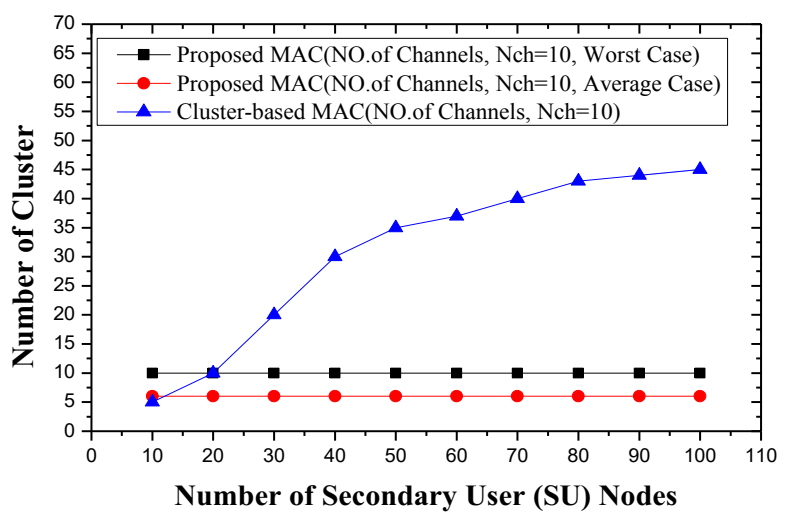

Fig. 6. Number of cluster Vs. Number of SUs nodes

\subsection{Throughput performance}

Fig. 7 demonstrate the aggregated throughput of proposed MAC with the Cluster-based MAC protocols as the network traffic is increased. The throughput of our

Fig. 5. Inter-communication scenario 


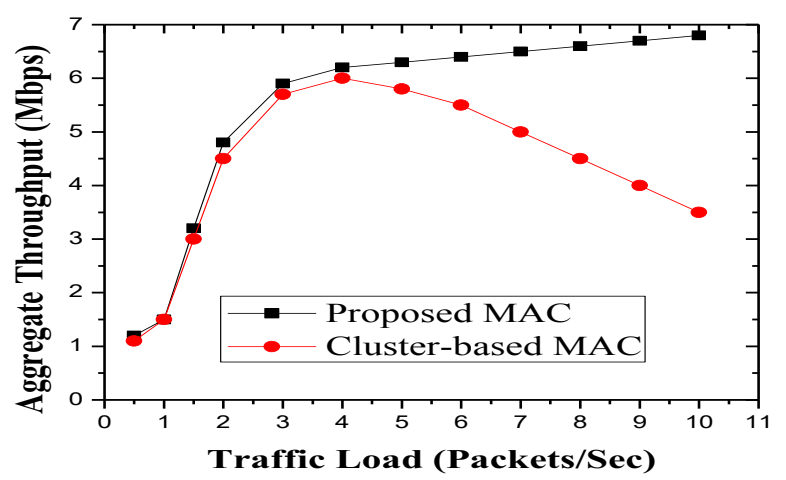

Fig. 7. Aggregated throughput Vs. traffic load

proposed MAC is significantly higher than Cluster-based MAC protocol. This is due to the fact that, Cluster-based MAC is CCC based solution, so bottleneck situation may occur in CCC. Therefore, throughput is decreased when network traffic is increased. In addition, inter cluster communication depends on the gateway nodes in Cluster-based MAC. Therefore, throughput may decrease lack of gateway node. On the other hand, our proposed protocol is channel id centric cluster based protocol, so communication takes place in the $1^{\text {st }}$ channel of the sorted $A C L$ list (i.e., CIC). Additionally, our proposed MAC does not depend on the CCC as well as gateway node during inter cluster communication. Therefore, throughput is increasing, when network traffic is increased.

\subsection{Packet transmission delay}

Fig. 8 demonstrate the packet transmission delay of proposed MAC with the Cluster-based MAC protocols as the network traffic is increased. The packet transmission deal of our proposed MAC is significantly lower than Cluster-based MAC protocol. This is due to the fact that, Cluster-based MAC is CCC based solution, so bottleneck situation may occur in CCC. Therefore, comparatively delay is increased when network traffic is increased. On the other hand, our proposed MAC is channel id centric cluster based protocol, so communication takes place in the $1^{\text {st }}$ channel of the sorted $A C L$ list (i.e., $C I C$ ). Additionally, our proposed

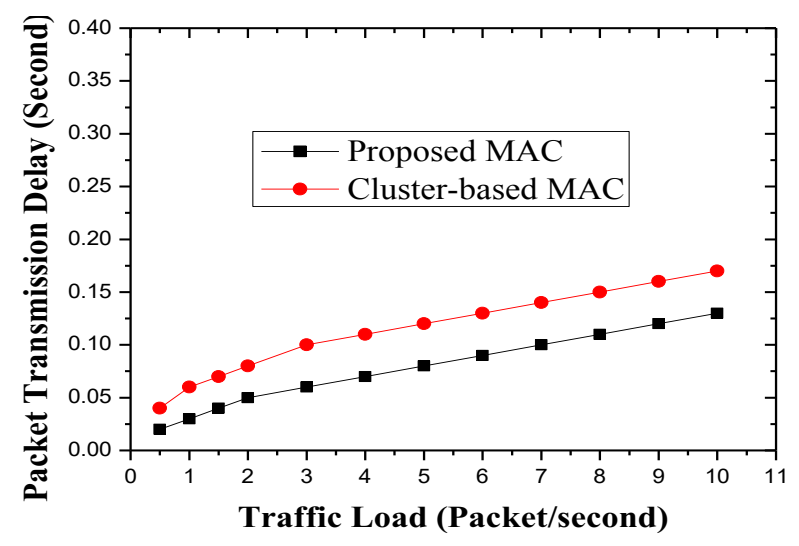

Fig. 8. Packet transmission delay Vs. traffic load
MAC does not depend on the CCC as well as gateway node during inter cluster communication. Therefore, comparatively delay is lower than Cluster-based. Moreover, due to more stable cluster structure proposed protocol has lower delay than Cluster-based MAC protocol.

\section{Conclusion}

In this paper we have presented a new cluster based MAC protocol for CRN using CIC based mechanism for SUs considering available channel list. The aim of proposed protocol is to create cluster structure more vigorous and use spectrum powerfully. Our proposed cluster formation algorithm is based on CIC and goals to maximize cluster throughput and maintain the cluster steadiness. Proposed MAC protocol algorithm ensures that maximum number of SUs can make channel rendezvous and successfully complete their data transmission with each other during intra and inter communication without gateway nodes(s). The simulation result show that proposed MAC can achieve lower number of clusters in the network, higher throughput, minimize packet transmission delay.

This work was collaboratively conducted by Universiti Malaysia Perlis (UniMAP) and by the Department of Computer Science and Engineering of Dhaka University of Engineering \& Technology (DUET), Gazipur, Bangladesh and Dr. M. O. Rahman is the corresponding author.

\section{References}

1. A. Hossain, S. Sultana, M.O. Rahman, Receiver Initiated Multi-channel Medium Access Control Protocol for Cognitive Radio Network, $2^{\text {nd }}$ International Conference on Electrical Information and Communication Technology (EICT), 350355(2015)

2. C. Ghosh, S. Roy, D. Cavalcanti, Coexistence challenges for heterogeneous cognitive wireless networks in TV white spaces, IEEE Wireless Communications Journal, 18, 22-31(2011)

3. J. Zhang, F. Yao, H. Zhao,Distributed Clustering in Cognitive Radio Ad Hoc Networks Using SoftConstraint Affinity Propagation, Journal of Radio engineering, 21, 785-794(2012)

4. N. Meghanathan, A survey on the communication protocols and security in cognitive radio networks, International Journal of Communication Networks and Information security (IJCNIS), 5, 19-38(2013)

5. I. F. Akyildiz, W. Y. Lee, M. C. vuran, S. Mohanty, Next generation dynamic spectrum access/cognitive radio wireless networks: a survey, Journal of Computer Networks, 50, 2127-2159(2006)

6. T. Chen, H. Zhang, G.M. Maggio, I. Chlamtac, CogMesh: A cluster-based cognitive radio network, In Proceedings of IEEE DySPAN, 168-178(2007)

7. X. Li, F. Hu, H. Zhang, X. Zhang, A Cluster-Based MAC Protocol for Cognitive Radio Ad Hoc 
Networks, Journal of Wireless Personal Communications, 69, 937-955(2013)

8. L. Lazos, S. Liu, M. Krunz, Spectrum OpportunityBased Control Channel Assignment in Cognitive Radio Networks,In Proceedings of IEEE $\operatorname{SECON}(2009)$

9. X. L. Huang, G. Wang, F. Hu, S. Kumar, Stabilitycapacity-adaptive routing for high mobility, multihop cognitive radio networks, IEEE Transactions on Vehicular Technology, 60, 2714-2729(2011)

10. K. E. Baddour, O. Ureten, T.J. Willink, A distributed message-passing approach for clustering cognitive radio networks, Journal of Wireless Personal Communications, 57, 119-133(2011)

11. R. Peeters, The maximum edge biclique problem is NP-complete, Journal of Discrete Applied Mathematics, 131, 651-654(2003)

12. W. Ye, J. Heidemann, D. Estrin, An energy effiecient MAC protocol for wireless sensor networks, Journal of Wireless Sensor Networks, 1, 59-69(2008) 